JOURNAL OF

APPLIED

CRYSTALLOGRAPHY

ISSN 1600-5767
₹ Present address: Advanced Institute of Materials Science, 2-6-8 Moniwadai, Taihaku, Sendai 982-0250, Japan.

Keywords: book reviews; multiscale materials modeling.

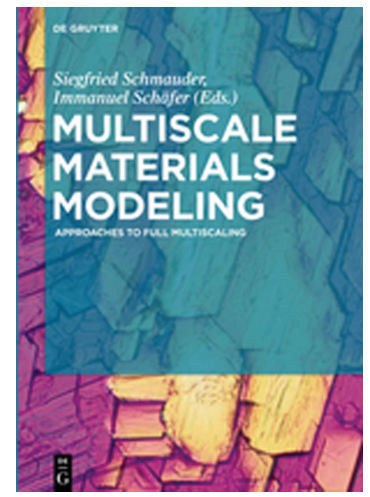

C 2018 International Union of Crystallography

\section{Multiscale Materials Modeling. Approaches to Full Multiscaling. Edited by Siegfried Schmauder and Immanuel Schäfer. De Gruyter, 2016. Pp. XX+326. Price (hardcover) EUR 119.95, USD 168.00, GBP 89.99. ISBN 978-3-11-041236-9.}

\author{
Yoshiaki Fukushima*¥
}

Comprehensive Research Organization for Science and Society, Center for Neutron Science, 162-1 Shirakata, Tokaimura, Nankagun, Ibaraki 319-1106, Japan. *Correspondence e-mail: y_fukushima@cross.or.jp

This book includes contributions from Stuttgart University members along with other experts from Germany (Interdisciplinary Center for Advanced Material Simulation, Max-Plank Institute for Metal Research and Friedrich-Alexander University) and other countries (Taiwan, Croatia, Ireland, Japan and Spain). All 16 chapters are easy to read and accessible not only to materials and computer scientists but also to non-specialized engineers and machine designers who seek an overview of the recent expansion in materials modeling. With the aim of providing a clear illustration of properties of materials such as fracture mechanism, the authors have tried to connect multiple simulation methods for the integrated description of behavior from the atomic scale to the macroscale.

About half the chapters in the book are concerned with iron and steel; in the other half, metals, oxide ceramics and organic polymers are also discussed. The focus is on the prediction of mechanical properties, such as deformation, fracture, fatigue and precipitation hardening, but there are also chapters devoted to magneto-electric and adsorbing properties. These review articles are provided in three parts, in which the topics of precipitation and strengthening effects (Part I), plastic deformation and fracture (Part II), and bio-inspired materials (Part III) are covered. The necessity for dividing the book into three parts is not clear and the chapters are remotely related to one another. The book is therefore suitable for those wishing to read individual chapters covering their own interests.

Fig. 1 shows a list of materials, the length range over which modeling methods were applied and target properties for the simulations.

As iron and steel are among the most important materials for our life and industry, modeling methods for their mechanical properties are covered in detail. Steel with high tensile strength, which is one of the most attractive materials, has a complicated microstructure with nano-size precipitation, and a multiscale treatment combining several modeling methods covering the nano-, micro- and millimetre length scales is necessary for its characterization. The influence of precipitation is discussed in the first two chapters. An overview of the modeling methods and their combinations from atomic $(\mathrm{nm})$ to practical $(\mathrm{m})$ scales is given in chapter 1, which is helpful for understanding the destinations of this book. Environments around the precipitation on nanometre to micrometre scales in steel (chapter 2), a Duralumin-type aluminium alloy (chapter 3) and ferroelectric ceramics (chapter 5) are explored by using Monte Carlo and molecular dynamics (MD) based modeling and analytical homogenization approaches. Problems of interfaces and atomic interactions in the interface regions are not discussed in these chapters, although the effects of interfaces are discussed for a simple bicrystal in chapters 4 and 7. The behavior of matrix phases is discussed in relation to dislocation movement in chapters 6 and 8 .

Methods for predicting macroscopic performance are discussed in Part II. Methods connected to the finite element method (FEM) are proposed for dealing with practical problems such as plastic deformation (chapter 8), fracture (chapter 9), fatigue of welded material (chapter 10), low-temperature brittleness (chapter 11) and behavior during cold 


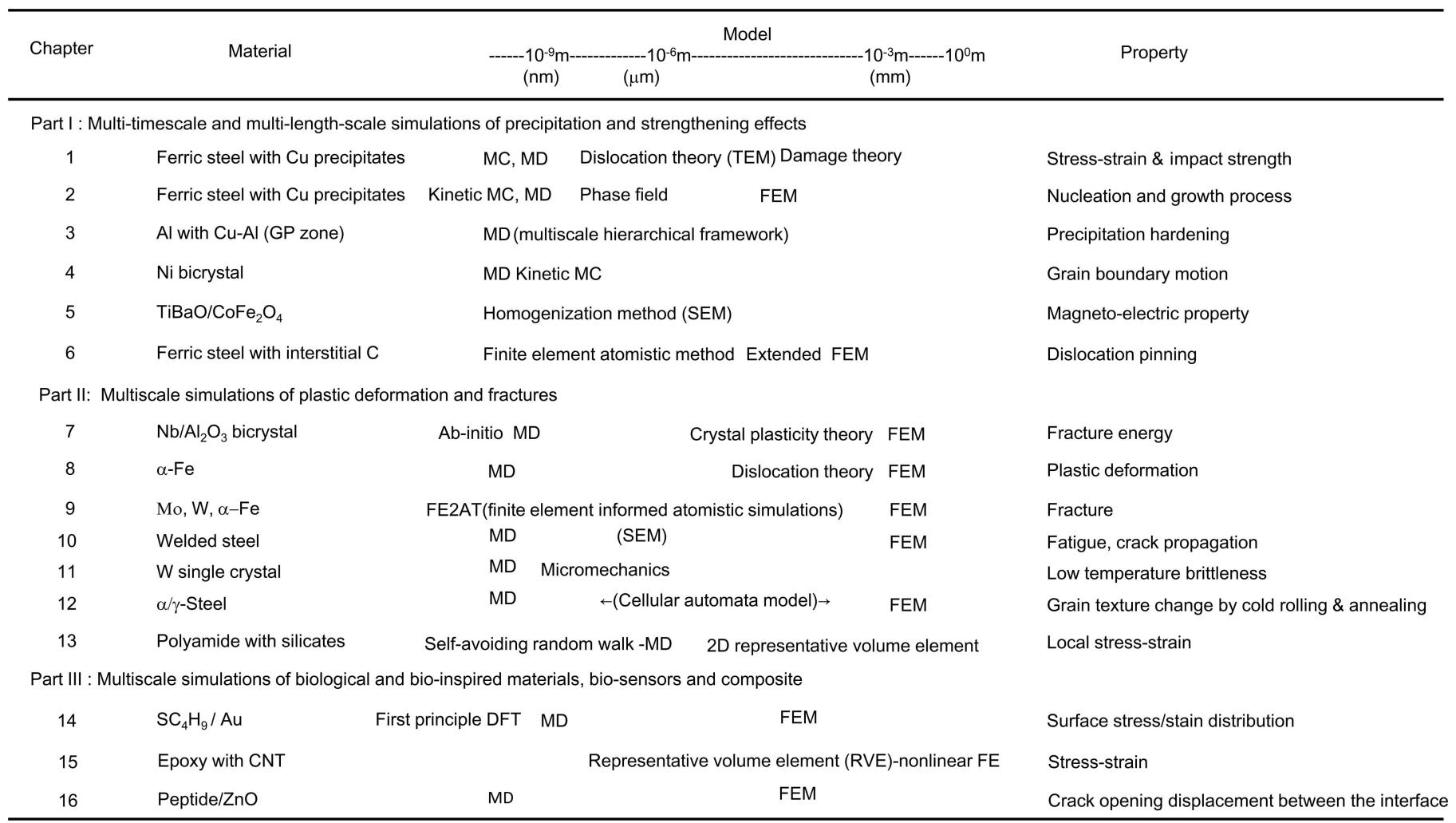

MD; molecular dynamics, MC; Monte Carlo simulation, FEM; finite element method, DFT; density functional theory

Figure 1

Materials, models and target properties covered in this book.

rolling processes (chapter 12). The authors propose unique approaches which would be useful for mechanical engineers and designers.

Organic polymers are the focus in chapters 13 and 15. A combined study of the arrangement of polyamide polymer chains on the nanometre scale near the silicate layers and local stress-strain properties by using modified MD and analytical methods is proposed in chapter 13. The macroscopic behavior at the micro- to millimetre scale in epoxy resin with carbon nanotubes is discussed from the viewpoint of the arrangement of the nanotube fibers by using a modified FEM method. Modeling approaches for soft matter are in the early stages of development.

In the last chapters in this book, the topic of interfaces is discussed again for $\mathrm{Au} /$ organic molecules in the nanometre region (chapter 14) and for oxide/peptides in the macroscopic region (chapter 16).

In most chapters, clear target properties which are important in recent industrial applications are shown and the modeling results are discussed with real experimental data. The enthusiasm of the computer scientists impressed me and gave recognition to the significance of multiscale modeling. Although good relations of computer science to mechanical engineering and manufacturing technology are apparent, the position with regard to solid or liquid state physics and chemistry or materials science is less clear.

The combination of modeling methods with experimental approaches to atomic interaction, interface structure and electromagnetic characterization is expected to be actively pursued in the future. 